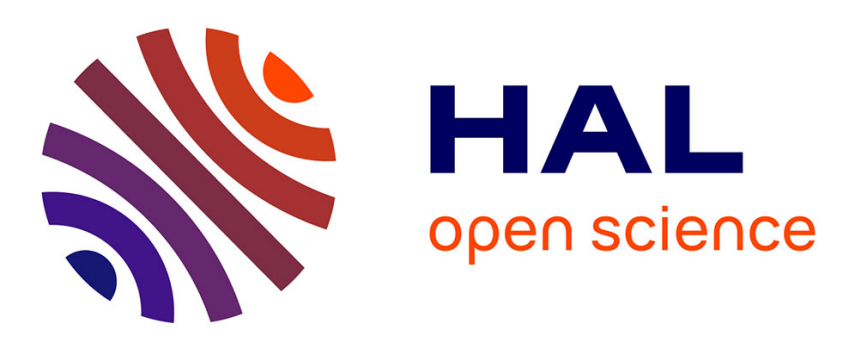

\title{
Congested traffic equilibria and degenerate anisotropic PDEs
}

\author{
Lorenzo Brasco, Guillaume Carlier
}

\section{To cite this version:}

Lorenzo Brasco, Guillaume Carlier. Congested traffic equilibria and degenerate anisotropic PDEs. 2012. hal-00734555

\section{HAL Id: hal-00734555 \\ https://hal.science/hal-00734555}

Preprint submitted on 23 Sep 2012

HAL is a multi-disciplinary open access archive for the deposit and dissemination of scientific research documents, whether they are published or not. The documents may come from teaching and research institutions in France or abroad, or from public or private research centers.
L'archive ouverte pluridisciplinaire HAL, est destinée au dépôt et à la diffusion de documents scientifiques de niveau recherche, publiés ou non, émanant des établissements d'enseignement et de recherche français ou étrangers, des laboratoires publics ou privés. 


\title{
Congested traffic equilibria and degenerate anisotropic PDEs
}

\author{
L. Brasco, G. Carlier ${ }^{\dagger}$
}

September 18, 2012

\begin{abstract}
Congested traffic problems on very dense networks lead, at the limit, to minimization problems posed on measures on curves as shown in [2]. Here, we go one step further by showing that these problems can be reformulated in terms of the minimization of an integral functional over a set of vector fields with prescribed divergence. We prove a Sobolev regularity result for their minimizers despite the fact that the Euler-Lagrange equation of the dual is highly degenerate and anisotropic. This somehow extends the analysis of [7] to the anisotropic case.
\end{abstract}

Keywords: traffic congestion, anisotropic and degenerate PDEs, regularity.

\section{Introduction}

Congested traffic equilibrium models on finite networks have received a lot of attention since the early 50's because of applications to road traffic as well as to communication networks. In such problems, the notion of Wardrop equilibrium [19] plays a distinguished role. The standard congested network model consists of:

- a finite oriented connected graph $G=\{N, E\}$ ( $N$ is the set of nodes of $G$ and $E$ the set of its edges) modelling the network;

- distribution of sources $f_{-}^{G}:=\sum_{x \in N} f_{-}^{G}(x) \delta_{x}$ and sinks $f_{+}^{G}:=\sum_{y \in N} f_{+}^{G}(y) \delta_{y}$ which are discrete measures with same total mass (which can be assumed to be 1) on the set of nodes $N$ that capture respectively the amount of traffic emitted and absorbed at the nodes of the network;

* LATP, Aix-Marseille Université, 13453, Marseille, FRANCE brasco@cmi.univ-mrs.fr

${ }^{\dagger}$ CEREMADE, UMR CNRS 7534, Université Paris IX Dauphine, 75775, Paris, FRANCE carlier@ceremade.dauphine.fr 
- for each edge $e \in E$, there is a function $h_{e}: \mathbb{R}_{+} \rightarrow \mathbb{R}_{+}$that is typically increasing and which captures the congestion effect in the sense that $h_{e}(m)$ represents the travelling time of edge $e$ when the traffic flow on $e$ is $m$.

One then looks for an equilibrium flow configuration, that is a flow configuration that is compatible with the distribution of sources and sinks and such that paths on which there is a positive flow minimize the travelling time between their endpoints, given the congestion effects. More precisely, a flow configuration is a probability measure $Q$ on the set $C^{G}$ of (loop-free) paths on the network that satisfies the mass conservation constraints

$$
\begin{aligned}
Q\left(\left\{\gamma \in C^{G}: \gamma \text { starts at } x\right\}\right) & =f_{-}^{G}(x), & & \text { for every } x \in N, \\
Q\left(\left\{\gamma \in C^{G}: \gamma \text { ends at } y\right\}\right) & =f_{+}^{G}(y), & & \text { for every } y \in N .
\end{aligned}
$$

A flow configuration $Q$ induces a collection of arc-flows through

$$
m^{Q}(e):=Q\left(\left\{\gamma \in C^{G}: e \in \gamma\right\}\right), \quad \text { for every } e \in E,
$$

and then a collection of arc-travelling times $h_{e}\left(m^{Q}(e)\right)$ as well as the total travelling time of a path $\gamma \in C^{G}$ :

$$
T^{Q}(\gamma)=\sum_{e \in \gamma} h_{e}\left(m^{Q}(e)\right)
$$

A Wardrop equilibrium is then by definition a flow configuration $Q$ such that for every $x$ and $y$ and every $\gamma$ in the set of paths $C_{x, y}^{G}$ starting at $x$ and ending at $y$, if $Q(\{\gamma\})>0$ then one shoud have

$$
T^{Q}(\gamma)=\min _{\tau \in C_{x, y}^{G}} T^{Q}(\tau)
$$

In other words, a Wardrop equilibrium requires that users behave rationally by choosing the shortest available paths, taking congestion into account, i.e. the fact that travel times increase with the flow. Finding Wardrop equilibria is a fixedpoint problem in nature that presents some analogies with the mean-field games theory of Lasry and Lions [15], eventhough it is purely stationary. Soon after the work of Wardrop, it was observed by Beckmann, McGuire and Winsten [4] that Wardrop equilibria coincide with the minimizers of the convex functional

$$
\sum_{e \in E} H_{e}\left(m^{Q}(e)\right) \quad \text { where } H_{e}(m)=\int_{0}^{m} h_{e}(s) d s,
$$

among flow configurations $Q$. This variational characterization is nice both from a theoretical point and for numerical computations. Unfortunately, the minimization problem above has one flow variable per admissible path on the network, it may 
therefore quickly become untractable for realistic road or communication networks. A natural question at this point is whether the situation somehow simplifies on very dense networks and if one can find some continuous counterpart to the discrete theory. This issue was recently addressed in [2] in the case of a two-dimensional cartesian grid, where $G=G^{\varepsilon}=\left\{N_{\varepsilon}, E_{\varepsilon}\right\}$ with

$$
N_{\varepsilon}=\varepsilon \mathbb{Z}^{2} \cap \bar{\Omega} \quad \text { and } \quad E_{\varepsilon}=\left\{\left[x, x+\varepsilon v_{k}\right]: x \in N_{\varepsilon}, k \in\{1, \ldots, 4\}\right\},
$$

where $\left(v_{1}, v_{2}, v_{3}, v_{4}\right):=((1,0),(0,1),(-1,0),(0,-1))$ denote the directions of the grid and $\Omega$ is some bounded two-dimensional domain. Under suitable assumptions ${ }^{1}$, it is shown in [2] that Wardrop equilibria at scale $\varepsilon$ converge as $\varepsilon \rightarrow 0^{+}$to solutions of

$$
\inf _{Q \in \mathcal{Q}\left(f_{+}, f_{-}\right)} \int_{\Omega} \sum_{k=1}^{4} H_{k}\left(x, m_{k}^{Q}(x)\right) d x
$$

where $\mathcal{Q}\left(f_{+}, f_{-}\right)$is the set of Borel probability measures $Q$ on $C\left([0,1], \mathbb{R}^{2}\right)$ satisfying the mass conservation conditions

$$
e_{0 \#} Q=f_{-}, \quad e_{1 \#} Q=f_{+}, \quad \text { where } e_{t}(\gamma)=\gamma(t), t \in[0,1],
$$

and such that $Q(\mathcal{L})=1$. Here $\mathcal{L}=W^{1, \infty}([0,1], \bar{\Omega})$ is the set of Lipschitz curves. For $k=1, \ldots, 4$, the measure $m_{k}^{Q}$ is defined by:

$$
\int_{\bar{\Omega}} \varphi(x) d m_{k}^{Q}(x):=\int_{\mathcal{L}}\left(\int_{0}^{1} \varphi(\gamma(t))\left(\dot{\gamma}(t) \cdot v_{k}\right)_{+} d t\right) d Q(\gamma)
$$

for every $\varphi \in C(\bar{\Omega}, \mathbb{R})$, while the functions $H_{k}$ are related to $h_{k}$ by $H_{k}(x, m)=$ $\int_{0}^{m} h_{k}(x, s) d s$. They are therefore convex and nondecreasing in their second argument, since $h_{k}$ are typically nonnegative and nondecreasing in their second argument. We shall suppose in the sequel that there is some $p>1$ such that for $k=1, \ldots, 4$, the functions $H_{k}$ satisfies the growth condition

$$
\frac{1}{\lambda}\left(m^{p}-1\right) \leq H_{k}(x, m) \leq \lambda\left(m^{p}+1\right) .
$$

So that in (1.1), it is intended that $m_{k}^{Q} \in L^{p}(\Omega), k=1, \ldots, 4$ and thus, we shall also assume that

$$
\mathcal{Q}_{p}\left(f_{+}, f_{-}\right):=\left\{Q \in \mathcal{Q}\left(f_{+}, f_{-}\right): m_{k}^{Q} \in L^{p}(\Omega), k=1, \ldots, 4\right\} \neq \emptyset .
$$

\footnotetext{
${ }^{1}$ In particular, the fact that the travelling time functions on $\operatorname{arcs}\left[x, x+\varepsilon v_{k}\right]$ scale like $\varepsilon h_{k}(x, m / \varepsilon)$ and that the discrete measures $f_{+}^{G_{\varepsilon}}$ and $f_{-}^{G_{\varepsilon}}$ weakly converge to some $f_{+}$and $f_{-}$.
} 
This assumption is satisfied for instance as soon as $f_{+}$and $f_{-}$belong to $L^{p}(\Omega)$ and $\Omega$ is convex, as follows from the results of De Pascale, Evans and Pratelli $[10,11,12]$ and Santambrogio [18].

Let us note that (1.1) is very similar to the optimal transport with congestion studied in [8], except that it keeps track of the anisotropy of the network. However, at first glance, it is absolutely not clear that problem (1.1) which is posed over probability measures over curves (two layers of infinite-dimensions!) offers any simplication with respect to the discrete problem. In the present paper, we want to go one step further with respect to the convergence results of [2] and we shall see that solving (1.1) roughly amounts to solve a single PDE. Following a similar approach as the one developed in [7] for the isotropic case, we shall investigate the relationship between (1.1) and the simpler problem

$$
\inf _{\sigma \in L^{p}\left(\Omega, \mathbb{R}^{2}\right)}\left\{\int_{\Omega} \sum_{k=1}^{4} H_{k}\left(x,\left(\sigma(x) \cdot v_{k}\right)_{+}\right) d x:-\operatorname{div} \sigma=f\right\},
$$

where $f:=f_{+}-f_{-}$and the equation $-\operatorname{div}(\sigma)=f$ should be understood as

$$
\int_{\Omega} \nabla u \cdot \sigma=\int_{\Omega} u d f, \quad \text { for every } u \in C^{1}(\bar{\Omega})
$$

so that it incorporates in the weak sense the homogeneous Neumann boundary condition $\sigma \cdot \nu_{\Omega}=0$ on $\partial \Omega$. Problem (1.6) belongs to a class of problems introduced by Beckmann [4] for the design of an efficient commodity transport program ${ }^{2}$. Solving (1.6) can be done by first solving the Euler-Lagrange equation of its dual formulation and then by using the primal-dual optimality conditions. However, in typical congestion models, the functions $H_{k}(x, \cdot)$ have a positive derivative at zero. Indeed, recall that this derivative is $h_{k}(x, 0)$ and it should be positive since one cannot go at infinite speed even when there is no congestion. This creates a singularity in the integrand in (1.6) which, in turn, makes the Euler-Lagrange equation of the dual extremely degenerate. Because of the anisotropic feature of the problem, the kind of degeneracies we are facing is even worse than the one in [7]. Indeed, the prototypical equation of [7] was

$$
-\operatorname{div}\left((|\nabla u|-1)_{+}^{q-1} \frac{\nabla u}{|\nabla u|}\right)=f
$$

with $q=p^{\prime}=p /(p-1)$, whereas here we shall rather deal with anisotropic equations of the form

$$
-\sum_{i=1}^{2} \partial_{i}\left(\left(\left|\partial_{i} u\right|-\delta_{i}\right)_{+}^{q-1} \frac{\partial_{i} u}{\left|\partial_{i} u\right|}\right)=f
$$

\footnotetext{
${ }^{2}$ Interestingly, the connection with the Monge-Kantorovich theory was realized much later by Robert McCann.
} 
which, in contrast with the isotropic case, degenerates in an unbounded set of values of the gradient. Even in the less degenerate case where all the $\delta_{i}$ 's are zero, the previous equation (refered to as the pseudo q-Laplacian equation in [5]) is more delicate than its isotropic counterpart, which has been much more studied and for which more regularity results are available.

The paper is organized as follows. In Section 2, we investigate some relationship between (1.1) and (1.6) in a similar way as in [7]. In Section 3, we formulate the optimality conditions for (1.6) in terms of the solutions of the dual and emphasize the kind of PDEs realistic anisotropic models of congestion lead to. Section 4 is devoted to some regularity results for such degenerate anisotropic elliptic PDEs. In this final section, we shall work in any dimension $d$.

\section{Relationship with Beckmann problem}

We consider again problems (1.1) and (1.6), our aim is to emphasize some precise connections between those two problems. We assume that $\Omega$ is an open bounded connected subset of $\mathbb{R}^{2}$ with a Lipschitz boundary, that (1.5) holds and that there is a neighbourhood $U$ of $\bar{\Omega}$ such that each function $H_{k}$ is Carathéodory on $U \times \mathbb{R}_{+}$, convex nondecreasing in its second argument with $H_{k}(x, 0)=0$ a.e. $x \in U$ and satisfies for some $\lambda>1$ the growth condition (1.4), for a.e. $x \in U$ and every $m \in \mathbb{R}_{+}$.

For $Q \in \mathcal{Q}_{p}\left(f_{+}, f_{-}\right)$, let us define the vector-measure $\sigma^{Q}$ by:

$$
\int_{\bar{\Omega}} F d \sigma^{Q}:=\int_{\mathcal{L}}\left(\int_{0}^{1} F(\gamma(t)) \cdot \dot{\gamma}(t) d t\right) d Q(\gamma), \quad \text { for every } F \in C\left(\bar{\Omega}, \mathbb{R}^{2}\right),
$$

where we recall that $\mathcal{L}=W^{1, \infty}([0,1], \bar{\Omega})$ has full mass for $Q$. For $u \in C^{1}(\bar{\Omega})$, we then have by the very definition of $\mathcal{Q}\left(f_{+}, f_{-}\right)$

$$
\int_{\bar{\Omega}} \nabla u \cdot d \sigma^{Q}=\int_{\mathcal{L}}(u(\gamma(1))-u(\gamma(0))) d Q(\gamma)=\int_{\bar{\Omega}} u d f
$$

i.e. $-\operatorname{div} \sigma^{Q}=f:=f_{+}-f_{-}$. Now for $\varphi \in C(\bar{\Omega})$ with $\varphi \geq 0$, let us observe that

$$
\begin{aligned}
\int_{\bar{\Omega}} \varphi d\left(\sigma^{Q} \cdot v_{k}\right) & =\int_{\mathcal{L}}\left(\int_{0}^{1} \varphi(\gamma(t)) \dot{\gamma}(t) \cdot v_{k} d t\right) d Q(\gamma) \\
& \leq \int_{\mathcal{L}}\left(\int_{0}^{1} \varphi(\gamma(t))\left(\dot{\gamma}(t) \cdot v_{k}\right)_{+} d t\right) d Q(\gamma)=\int_{\bar{\Omega}} \varphi d m_{k}^{Q},
\end{aligned}
$$

i.e. for $k=1, \ldots, 4$, one has $\sigma^{Q} \cdot v_{k} \leq m_{k}^{Q}$ in the sense of measures. Since $Q \in \mathcal{Q}_{p}\left(f_{+}, f_{-}\right)$, this implies that $\sigma^{Q} \in L^{p}\left(\Omega, \mathbb{R}^{2}\right)$ and thus

$$
\left(\sigma^{Q} \cdot v_{k}\right)_{+} \leq m_{k}^{Q} \quad \text { a.e. in } \Omega \text {. }
$$


Note then that the assumption (1.5) asserting that $\mathcal{Q}_{p}\left(f_{+}, f_{-}\right)$is not empty implies that $-\operatorname{div} \sigma=f$ can be solved in $L^{p}\left(\Omega, \mathbb{R}^{2}\right)$, so that the zero-mass signed measure $f$ belongs to the dual of $W^{1, q}(\Omega)$, where $q=p /(p-1)$ is the conjugate exponent of $p$. The existence of solutions to (1.6) directly follows; as for the existence of solutions to (1.1), it follows from the same arguments as in [2] or [8].

Theorem 2.1. Under the assumptions above, we have

$$
\inf (1.1)=\inf (1.6)
$$

Proof. Let $Q \in \mathcal{Q}_{p}\left(f_{+}, f_{-}\right)$, since $H_{k}$ is nondecreasing in its second argument, we deduce from (2.2) that

$$
\int_{\Omega} \sum_{k=1}^{4} H_{k}\left(x, m_{k}^{Q}\right) d x \geq \int_{\Omega} \sum_{k=1}^{4} H_{k}\left(x,\left(\sigma \cdot v_{k}\right)_{+}\right) d x,
$$

and since $-\operatorname{div}\left(\sigma^{Q}\right)=f:=f_{+}-f_{-}$, we immediately get inf $(1.1) \geq \inf (1.6)$.

For the converse inequality, we shall use Moser's flow argument (see [9, 17]), together with some standard regularization as follows. Let $\sigma$ be a solution of (1.6), extend it by 0 outside $\Omega$, let then $\rho \in C_{c}^{\infty}\left(\mathbb{R}^{2}\right)$ be a positive function, supported in the unit ball $B_{1}$ and such that $\int_{\mathbb{R}^{2}} \rho=1$. For $\varepsilon \ll 1$ so that $\Omega_{\varepsilon}:=\Omega+\varepsilon B_{1} \Subset U$, we set $\rho_{\varepsilon}(x):=\varepsilon^{-2} \rho\left(\varepsilon^{-1} x\right)$ and $\sigma^{\varepsilon}:=\rho_{\varepsilon} * \sigma$. By construction, we thus have that $\sigma^{\varepsilon} \in C^{\infty}\left(\bar{\Omega}_{\varepsilon}\right)$ and

$$
-\operatorname{div} \sigma^{\varepsilon}=f_{+}^{\varepsilon}-f_{-}^{\varepsilon} \quad \text { in } \Omega_{\varepsilon}, \quad \sigma^{\varepsilon}=0, \text { on } \partial \Omega_{\varepsilon},
$$

where $f_{ \pm}^{\varepsilon}=\rho_{\varepsilon} *\left(f_{ \pm} 1_{\bar{\Omega}}\right)+\varepsilon$. Now comes Moser's flow construction: for $t \in[0,1]$ and $x \in \bar{\Omega}_{\varepsilon}$, we set $g^{\varepsilon}(t, x):=(1-t) f_{-}^{\varepsilon}(x)+t f_{+}^{\varepsilon}(x)$, since $\sigma^{\varepsilon}$ and $g^{\varepsilon}$ are smooth and the latter is bounded from below by $\varepsilon>0$, one may define the flow $X^{\varepsilon}$ of the vector field $v^{\varepsilon}:=\sigma^{\varepsilon} / g^{\varepsilon}$, i.e.

$$
\begin{aligned}
& \dot{X}_{t}^{\varepsilon}(x)=v^{\varepsilon}\left(t, X_{t}^{\varepsilon}(x)\right)=\frac{\sigma^{\varepsilon}\left(X_{t}^{\varepsilon}(x)\right)}{(1-t) f_{-}^{\varepsilon}\left(X_{t}^{\varepsilon}(x)\right)+t f_{+}^{\varepsilon}\left(X_{t}^{\varepsilon}(x)\right)}, \\
& X_{0}^{\varepsilon}(x)=x, \quad(t, x) \in[0,1] \times \bar{\Omega}_{\varepsilon} .
\end{aligned}
$$

Since by construction $g^{\varepsilon}$ satisfies the continuity equation $\partial_{t} g^{\varepsilon}+\operatorname{div}\left(g^{\varepsilon} v^{\varepsilon}\right)=0$, with initial datum $g^{\varepsilon}(0, \cdot)=f_{-}^{\varepsilon}$ and $v^{\varepsilon}$ is smooth, we have $X_{t \#}^{\varepsilon} f_{-}^{\varepsilon}=g^{\varepsilon}(t, \cdot)$ for every $t \in[0,1]$. Let then $\mathcal{L}_{\varepsilon}:=W^{1, \infty}\left([0,1], \bar{\Omega}_{\varepsilon}\right)$ and consider the measure $Q^{\varepsilon}$ on $\mathcal{L}_{\varepsilon}$ defined by

$$
Q^{\varepsilon}:=\int_{\bar{\Omega}_{\varepsilon}} \delta_{X^{\varepsilon}(x)} d f_{-}^{\varepsilon}(x)
$$


We then have $e_{t \#} Q^{\varepsilon}=X_{t \#}^{\varepsilon} f_{-}^{\varepsilon}=g^{\varepsilon}(t, \cdot)$ for every $t \in[0,1]$ and in particular

$$
e_{0 \#} Q^{\varepsilon}=f_{-}^{\varepsilon} \quad \text { and } \quad e_{1 \#} Q^{\varepsilon}=f_{+}^{\varepsilon} \text {. }
$$

Now let us define $\sigma^{Q^{\varepsilon}}$ and $m_{k}^{Q^{\varepsilon}}$ as in (2.1) and (1.3) respectively, by using testfunctions that are now defined on $\bar{\Omega}_{\varepsilon}$. For every $\varphi \in C\left(\bar{\Omega}_{\varepsilon}\right)$, by the definition of $Q^{\varepsilon}$, the fact that $X_{t \#}^{\varepsilon} f_{-}^{\varepsilon}=g^{\varepsilon}(t, \cdot)$ and that $v^{\varepsilon} g^{\varepsilon}=\sigma^{\varepsilon}$ and by using Fubini's formula, we then have

$$
\begin{aligned}
\int_{\bar{\Omega}_{\varepsilon}} \varphi d m_{k}^{Q^{\varepsilon}} & =\int_{\bar{\Omega}_{\varepsilon}}\left(\int_{0}^{1} \varphi\left(X_{t}^{\varepsilon}(x)\right)\left(v^{\varepsilon}\left(t, X_{t}^{\varepsilon}(x)\right) \cdot v_{k}\right)_{+} d t\right) d f_{-}^{\varepsilon}(x) \\
& =\int_{0}^{1}\left(\int_{\bar{\Omega}_{\varepsilon}} \varphi(y)\left(v^{\varepsilon}(t, y) \cdot v_{k}\right)_{+} d\left(X_{t \#}^{\varepsilon} f_{-}^{\varepsilon}\right)(y)\right) d t \\
& =\int_{0}^{1}\left(\int_{\bar{\Omega}_{\varepsilon}} \varphi(y)\left(v^{\varepsilon}(t, y) \cdot v_{k}\right)_{+} g^{\varepsilon}(t, y) d y\right) d t \\
& =\int_{\bar{\Omega}_{\varepsilon}} \varphi(y)\left(\sigma^{\varepsilon}(y) \cdot v_{k}\right)_{+} d y
\end{aligned}
$$

so that $m_{k}^{Q^{\varepsilon}}=\left(\sigma^{\varepsilon} \cdot v_{k}\right)_{+}$. A similar computation (with a vector valued test-function) similarly shows that $\sigma^{Q^{\varepsilon}}=\sigma^{\varepsilon}$.

Now, let us remark that the definitions of $m_{k}^{Q}$ and $\sigma^{Q}$ are invariant by arbitrary injective reparameterization of curves. In particular, if for any Lipischitz curve $\gamma$, we denote by $\widetilde{\gamma}$ its constant speed reparametrization and if we denote by $\widetilde{Q}$ the push forward of $Q$ through the map $\gamma \mapsto \widetilde{\gamma}$, we have $m_{k}^{\widetilde{Q}}=m_{k}^{Q}$ and $\sigma^{\widetilde{Q}}=\sigma^{Q}$. Arguing as in [8, Lemma 2.8], the uniform $L^{p}$ bound on $\sigma^{\widetilde{Q}^{\varepsilon}}=\sigma^{Q^{\varepsilon}}=\sigma^{\varepsilon}$ implies that the family of Borel measures $\widetilde{Q}^{\varepsilon}$ on $C\left([0,1], \mathbb{R}^{2}\right)$ is tight and thus admits a (not relabeled) subsequence that $*$-weakly converges to some measure $Q$. Observe that this limit $Q$ is a probability measure, since the total mass of $\widetilde{Q}^{\varepsilon}$ equals that of $f_{+}^{\varepsilon}$ i.e. $1+\varepsilon$ and, arguing again as in [8, Lemma 2.8], one can show that $Q(\mathcal{L})=1$ (more precisely for fixed $\varepsilon>0$, one proves exactly as in [8, Lemma 2.8] that $Q\left(\mathcal{L}_{\varepsilon}\right)=1$ so that $\left.Q(\mathcal{L})=\lim _{\varepsilon \downarrow 0^{+}} Q\left(\mathcal{L}_{\varepsilon}\right)=1\right)$. Moreover, thanks to the $*$-weak convergence of $\widetilde{Q}^{\varepsilon}$ to $Q$, passing to the limit in (2.4) gives $Q \in \mathcal{Q}\left(f_{+}, f_{-}\right)$. We finally remark that $m_{k}^{\widetilde{Q}^{\varepsilon}}=m_{k}^{Q^{\varepsilon}}=\left(\sigma^{\varepsilon} \cdot v_{k}\right)_{+}$converges strongly in $L^{p}$ to $\left(\sigma \cdot v_{k}\right)_{+}$ and then, thanks to the same semicontinuity argument as in [8, Lemma 2.9], we have $m_{k}^{Q} \leq\left(\sigma \cdot v_{k}\right)_{+}$in the sense of measures. This implies that $m_{k}^{Q} \in L^{p}$ i.e. $Q \in \mathcal{Q}_{p}\left(f_{+}, f_{-}\right)$. Using the monotonicity of $H_{k}$, we obtain

$$
\int_{\Omega} \sum_{k=1}^{4} H_{k}\left(x, m_{k}^{Q}(x)\right) d x \leq \int_{\Omega} \sum_{k=1}^{4} H_{k}\left(x,\left(\sigma(x) \cdot v_{k}\right)_{+}\right) d x=\inf (1.6)
$$

and thus we can finally infer $\inf (1.1) \leq \inf (1.6)$. 
Remark 2.2. The convexity assumption on the $H_{k}$ 's is essential for the existence of minimizers, but it played no role in the proof that inf $(1.1)=\inf (1.6)$ where only monotonicity matters.

Remark 2.3. Note that with the previous proof, we have in fact also shown slightly more precise results. Firstly

$$
Q \text { solves }(1.1) \Longrightarrow \sigma^{Q} \text { solves (1.6). }
$$

Secondly, if in addition each function $H_{k}$ is increasing in its second argument, it easily follows from inequality (2.2) that we have the equivalence

$$
Q \text { solves }(1.1) \Longleftrightarrow \sigma^{Q} \text { solves }(1.6) \text { and } m_{k}^{Q}=\left(\sigma^{Q} \cdot v_{k}\right)_{+}, k=1, \ldots, 4 \text {. }
$$

Lastly, we have also shown how to build a minimizing sequence for (1.1) from a regularization of a solution $\sigma$ of (1.6) thanks to Moser's flow method. Also notice that if $\sigma$ and $f_{+}$and $f_{-}$are regular enough, so that the flow can be defined (in the Ambrosio-Di Perna-Lions sense [1, 13], for instance), there is no need to do such a regularization. By the way, the regularity of $\sigma$ will be addressed in section 4 .

\section{$3 \quad$ Link with anisotropic elliptic PDEs}

To shorten notations, let us set

$$
\mathcal{H}(x, \sigma):=\sum_{k=1}^{4} H_{k}\left(x,\left(\sigma \cdot v_{k}\right)_{+}\right),
$$

which can also be written in the separable form $\mathcal{H}(x, \sigma)=\mathcal{H}_{1}\left(x, \sigma_{1}\right)+\mathcal{H}_{2}\left(x, \sigma_{2}\right)$, where

$$
\mathcal{H}_{1}\left(x, \sigma_{1}\right)=H_{1}\left(x, \sigma_{1+}\right)+H_{3}\left(x, \sigma_{1-}\right), \quad \mathcal{H}_{2}\left(x, \sigma_{2}\right)=H_{2}\left(x, \sigma_{2+}\right)+H_{4}\left(x, \sigma_{2-}\right) .
$$

The dual problem of (1.6) then reads

$$
\sup _{u \in W^{1, q}(\Omega)}\left\{\int_{\bar{\Omega}} u d f-\int_{\Omega} \mathcal{H}^{*}(x, \nabla u) d x\right\},
$$

and since $f=f_{+}-f_{-}$has zero mean, we may as well restrict the optimization in (3.2) to zero-mean $W^{1, q}(\Omega)$ functions. Here $q=p^{\prime}=p /(p-1)$. As usual, $\mathcal{H}^{*}(x, \cdot)$ denotes the Legendre transform of $\mathcal{H}(x, \cdot)$ and it is separable as well $\mathcal{H}^{*}(x, z)=\mathcal{H}_{1}^{*}\left(x, z_{1}\right)+\mathcal{H}_{2}^{*}\left(x, z_{2}\right)$.

Let us recall that in congestion models the functions $H_{k}(x, \cdot)$ are the primitives of functions $h_{k}(x, \cdot)$, where $h_{k}(x, m)$ represents the time per unit of length to move 
at $x$ in the direction $v_{k}$ when the intensity of traffic in this direction is $m$. The functions $h_{k}$ therefore represent a sort of anisotropic metric that captures the congestion effect through some relationship between time per unit of length and mass per unit of length. Having this interpretation in mind, it is reasonable to assume that $h_{k}(x, \cdot)$ are continuous increasing functions on $\mathbb{R}_{+}$which satisfy some $p-1$ growth condition (so that (1.4) holds) and that $h_{k}(x, 0)>0$ (the metric is everywhere positive even when there is no traffic). This implies that $\mathcal{H}$ is strictly convex in its second variable (hence, by duality $\mathcal{H}^{*}$ is $C^{1}$ ) but not differentiable on the axes of coordinates, more precisely, the subdifferentials of $\mathcal{H}_{1}(x,$.$) and$ $\mathcal{H}_{2}(x,$.$) are intervals having 0$ in their interior. The Legendre transforms $\mathcal{H}_{1}^{*}(x,$. and $\mathcal{H}_{2}^{*}(x,$.$) therefore vanish on such intervals which makes the Euler-Lagrange$ equation of (3.2) degenerate close to the axes of coordinates.

Recall that by standard convex duality (see [14] for example), the values $\min (1.6)$ and $\max (3.2)$ coincide and the primal-dual optimality conditions characterize the minimizer (unique by strict convexity) $\sigma$ of (1.6) by

$$
\sigma(x)=\nabla \mathcal{H}^{*}(x, \nabla u(x)), \quad \text { a.e. } x \in \Omega
$$

where $u$ is a solution of (3.2). This is equivalent to the requirement that $u$ is a weak solution of the Euler-Lagrange equation:

$$
-\operatorname{div} \nabla \mathcal{H}^{*}(x, \nabla u)=f, \quad \text { in } \Omega, \quad \nabla \mathcal{H}^{*}(x, \nabla u) \cdot \nu_{\Omega}=0, \quad \text { on } \partial \Omega,
$$

in the sense that

$$
\int_{\Omega} \nabla \mathcal{H}^{*}(x, \nabla u(x)) \cdot \nabla \varphi(x) d x=\int_{\bar{\Omega}} \varphi(x) d f(x), \quad \text { for every } \varphi \in W^{1, q}(\Omega) .
$$

Let us note that eventhough $u$ is non unique, $\sigma$ is.

A prototypical example is $h_{k}(x, m)=a_{k}(x) m^{p-1}+\delta_{k}$ with $\delta_{k}>0$ and the weights $a_{k}$ are smooth and bounded away from zero. A direct computation then gives

$$
\mathcal{H}_{1}^{*}\left(x, z_{1}\right)=\frac{b_{1}(x)}{q}\left(z_{1}-\delta_{1}\right)_{+}^{q}+\frac{b_{3}(x)}{q}\left(-z_{1}-\delta_{3}\right)_{+}^{q},
$$

and

$$
\mathcal{H}_{2}^{*}\left(x, z_{2}\right)=\frac{b_{2}(x)}{q}\left(z_{2}-\delta_{2}\right)_{+}^{q}+\frac{b_{4}(x)}{q}\left(-z_{2}-\delta_{4}\right)_{+}^{q},
$$

where $b_{k}=a_{k}^{-\frac{1}{p-1}}$. In this case (3.3) takes the form

$$
\begin{aligned}
& -\partial_{1}\left(b_{1}\left(\partial_{1} u-\delta_{1}\right)_{+}^{q-1}-b_{3}\left(-\partial_{1} u-\delta_{3}\right)_{+}^{q-1}\right) \\
& -\partial_{2}\left(b_{2}\left(\partial_{2} u-\delta_{2}\right)_{+}^{q-1}-b_{4}\left(-\partial_{2} u-\delta_{4}\right)_{+}^{q-1}\right)=f .
\end{aligned}
$$


Observe that in the symmetric homogeneous case, i.e. when $\delta_{1}=\delta_{3}, \delta_{2}=\delta_{4}$ and $a_{k} \equiv 1$, the previous simplifies to

$$
-\partial_{1}\left(\left(\left|\partial_{1} u\right|-\delta_{1}\right)_{+}^{q-1} \frac{\partial_{1} u}{\left|\partial_{1} u\right|}\right)-\partial_{2}\left(\left(\left|\partial_{2} u\right|-\delta_{2}\right)_{+}^{q-1} \frac{\partial_{2} u}{\left|\partial_{2} u\right|}\right)=f .
$$

Since $\mathcal{H}_{1}^{*}\left(x, z_{1}\right)$ and $\mathcal{H}_{2}^{*}\left(x, z_{2}\right)$ vanish whenever $z_{1} \in\left[-\delta_{3}, \delta_{1}\right]$ and $z_{2} \in\left[-\delta_{4}, \delta_{2}\right]$ respectively, any $u$ whose gradient belongs to the rectangle $\left[-\delta_{3}, \delta_{1}\right] \times\left[-\delta_{4}, \delta_{2}\right]$ solves the previous equation with $f=0$. Hence, there is no hope to recover estimates on the second derivatives of $u$ or even oscillation estimates on $\nabla u$ from (3.4). The best one can hope for is that $u$ is Lipschitz. However, we shall see in the next section how to obtain some regularity results directly for the vector field $\sigma=\left(\sigma_{1}, \sigma_{2}\right)$ that solves (1.6), i.e.

$$
\begin{aligned}
& \sigma_{1}=b_{1}\left(\partial_{1} u-\delta_{1}\right)_{+}^{q-1}-b_{3}\left(-\partial_{1} u-\delta_{3}\right)_{+}^{q-1}, \\
& \sigma_{2}=b_{2}\left(\partial_{2} u-\delta_{2}\right)_{+}^{q-1}-b_{4}\left(-\partial_{2} u-\delta_{4}\right)_{+}^{q-1} .
\end{aligned}
$$

Typical traffic congestion problems are two-dimensional, however degenerate equations of the form (3.4) may arise in other contexts, as in the relaxation of some nonconvex variational problems. For this reason, in the next section we will actually work in arbitrary dimension $d$.

\section{Regularity}

\subsection{A general result}

Let $q \geq 2, \Omega$ be an open bounded subset of $\mathbb{R}^{d}, F: \Omega \times \mathbb{R}^{d} \rightarrow \mathbb{R}^{d}$ and $G: \mathbb{R}^{d} \rightarrow \mathbb{R}^{d}$ be some continuous vector fields such that there exist $\nu>0$ and $\mu \geq 0$ such that for every $(x, z)$ and $(y, w)$ in $\Omega \times \mathbb{R}^{d}$, one has

$$
\begin{gathered}
|F(x, z)| \leq \mu|z|^{q-1}, \\
(F(x, z)-F(x, w)) \cdot(z-w) \geq \nu|G(z)-G(w)|^{2}, \\
|F(x, z)-F(x, w)| \leq \mu\left(|G(z)|^{\frac{q-2}{q}}+|G(w)|^{\frac{q-2}{q}}\right)|G(z)-G(w)|,
\end{gathered}
$$

$F(\cdot, z)$ is differentiable for every $z$ and satisfies

$$
\begin{gathered}
\left|\nabla_{x} F(x, z)\right| \leq \mu|G(z)|^{\frac{2 q-2}{q}} \\
\left|\nabla_{x} F(x, z)-\nabla_{x} F(x, w)\right| \leq \mu\left(|G(z)|^{\frac{q-2}{q}}+|G(q)|^{\frac{q-2}{q}}\right)|G(z)-G(w)| \\
\left|\nabla_{x} F(x, z)-\nabla_{x} F(y, z)\right| \leq \mu|x-y||G(z)|^{\frac{2 q-2}{q}}
\end{gathered}
$$


Let finally $f \in W_{\text {loc }}^{1, p}(\Omega)$ with $p=q^{\prime}$ and let us consider the equation

$$
-\operatorname{div} F(x, \nabla u)=f .
$$

Using Nirenberg's method of incremental ratios, we then get the following result.

Theorem 4.1. Let $q \geq 2, p=q^{\prime}, f \in W_{\mathrm{loc}}^{1, p}(\Omega), F$ and $G$ be vector fields that satisfy conditions (4.1)-(4.6) above and let $u \in W_{\mathrm{loc}}^{1, q}(\Omega)$ be a local weak solution of (4.7). Then $\mathcal{G}:=G(\nabla u) \in W_{\mathrm{loc}}^{1,2}(\Omega)$.

Proof. To shorten notations, set $\mathcal{F}:=F\left(\cdot, \nabla u(\cdot)\right.$ ) (note that $\mathcal{F} \in L_{\text {loc }}^{p}(\Omega)$ and $\mathcal{G} \in L_{\text {loc }}^{2}(\Omega)$ thanks to $\left.(4.1)\right)$ and denote by $\tau_{h} \varphi:=\varphi(\cdot+h)$ the translate of the function $\varphi$ by the vector $h$. Let $\varphi \in W^{1, p}(\Omega)$ be compactly supported in $\Omega$ and $h \in \mathbb{R}^{d} \backslash\{0\}$ be such that $|h|<\operatorname{dist}\left(\operatorname{supp}(\varphi), \mathbb{R}^{d} \backslash \Omega\right)$, we then have

$$
\int_{\Omega} \frac{\tau_{h} \mathcal{F}-\mathcal{F}}{|h|} \cdot \nabla \varphi d x=\int_{\Omega} \frac{\tau_{h} f-f}{|h|} \varphi d x
$$

Let $\omega \Subset \omega_{0} \Subset \Omega$, let $\xi \in C_{c}^{\infty}(\Omega)$ be such that $\operatorname{supp}(\xi) \subset \omega_{0}, 0 \leq \xi \leq 1$ and $\xi=1$ on $\bar{\omega}$ and $h \in \mathbb{R}^{d} \backslash\{0\}$ such that $|h| \leq r_{0}<\frac{1}{2} \operatorname{dist}\left(\omega_{0}, \mathbb{R}^{d} \backslash \Omega\right)$. In what follows, $c$ will denote a nonnegative constant that does not depend on $h$, but may vary from one line to another. We then insert the test function

$$
\varphi=\xi^{2}|h|^{-1}\left(\tau_{h} u-u\right)
$$

into (4.8). Using the fact that $u \in W_{\text {loc }}^{1, q}(\Omega), f \in W_{\text {loc }}^{1, p}(\Omega)$ and Hölder's inequality and defining $\omega^{\prime}:=\omega_{0}+B\left(0, r_{0}\right)$, we get

$$
|h|^{-2} \int_{\Omega}\left(\tau_{h} \mathcal{F}-\mathcal{F}\right) \cdot\left(\xi^{2}\left(\tau_{h} \nabla u-\nabla u\right)+2 \xi \nabla \xi\left(\tau_{h} u-u\right)\right) \leq\|\nabla f\|_{L^{p}\left(\omega^{\prime}\right)}\|\nabla u\|_{L^{q}\left(\omega^{\prime}\right)} .
$$

Let us now write the left-hand side of the previous inequality as the sum of four terms $I_{1}+I_{2}+I_{3}+I_{4}$, where

$$
\begin{gathered}
I_{1}:=|h|^{-2} \int_{\Omega} \xi^{2}\left(F(x+h, \nabla u(x+h))-F(x+h, \nabla u(x)) \cdot\left(\tau_{h} \nabla u-\nabla u\right),\right. \\
I_{2}:=|h|^{-2} \int_{\Omega}\left(F(x+h, \nabla u(x+h))-F(x+h, \nabla u(x)) \cdot \nabla \xi \xi\left(\tau_{h} u-u\right),\right. \\
I_{3}:=|h|^{-2} \int_{\Omega}\left(F(x+h, \nabla u(x))-F(x, \nabla u(x)) \cdot \nabla \xi \xi\left(\tau_{h} u-u\right),\right.
\end{gathered}
$$

and

$$
I_{4}:=|h|^{-2} \int_{\Omega}\left(F(x+h, \nabla u(x))-F(x, \nabla u(x)) \cdot\left(\tau_{h} \nabla u-\nabla u\right) \xi^{2} .\right.
$$


Thanks to (4.2), the first term satisfies satisfies:

$$
I_{1} \geq \nu\left\|\xi|h|^{-1}\left(\tau_{h} \mathcal{G}-\mathcal{G}\right)\right\|_{L^{2}}^{2} .
$$

As for the second term, if $q>2$ using (4.3) and Hölder's inequality with exponents $2, q$ and $\frac{2 q}{q-2}$ yields

$$
\begin{aligned}
\left|I_{2}\right| & \leq|h|^{-2} \int_{\Omega}\left|\xi \nabla \xi \| \tau_{h} u-u\right|\left|\tau_{h} \mathcal{G}-\mathcal{G}\right|\left(\left|\tau_{h} \mathcal{G}\right|^{\frac{q-2}{q}}+|\mathcal{G}|^{\frac{q-2}{q}}\right) \\
& \leq c\|\nabla \xi\|_{L^{\infty}}\left\||h|^{-1}\left(\tau_{h} u-u\right)\right\|_{L^{q}\left(\omega_{0}\right)}\left\|\xi|h|^{-1}\left(\tau_{h} \mathcal{G}-\mathcal{G}\right)\right\|_{L^{2}}\left(\int_{\omega_{0}}|\mathcal{G}|^{2}+\left|\tau_{h} \mathcal{G}\right|^{2}\right)^{\frac{q-2}{2 q}} \\
& \leq c\left\|\xi|h|^{-1}\left(\tau_{h} \mathcal{G}-\mathcal{G}\right)\right\|_{L^{2}},
\end{aligned}
$$

and if $q=2$, simply using Cauchy-Schwarz inequality, we similarly obtain:

$$
\left|I_{2}\right| \leq c\left\|\xi|h|^{-1}\left(\tau_{h} \mathcal{G}-\mathcal{G}\right)\right\|_{L^{2}}
$$

We now come to the term $I_{3}$. Thanks to (4.4), we have

$$
|h|^{-1}|F(x+h, \nabla u(x))-F(x, \nabla u(x))| \leq \mu|\mathcal{G}|^{\frac{2 q-2}{q}} \in L_{\mathrm{loc}}^{p},
$$

using again Hölder's inequality and the fact that $\left\||h|^{-1}\left(\tau_{h} u-u\right)\right\|_{L^{q}\left(\omega_{0}\right)}$ is bounded independently of $h$, we get $\left|I_{3}\right| \leq C$. To handle the last term we first write

$$
\frac{F(x+h, \nabla u(x))-F(x, \nabla u(x))}{|h|}=\int_{0}^{1} \nabla_{x} F(x+s h, \nabla u(x)) \frac{h}{|h|} d s .
$$

By defining

$$
\Phi_{h}(x):=\int_{0}^{1} \frac{\nabla_{x} F(x+s h, \nabla u(x))-\nabla_{x} F(x+(1+s) h, \nabla u(x+h))}{|h|} \frac{h}{|h|} d s,
$$

and rearranging terms, we can rewrite

$$
\begin{aligned}
I_{4} & =\int_{\Omega} \xi^{2} \tau_{h} \nabla u \cdot \Phi_{h} d x \\
& -\int_{\Omega} \frac{\tau_{h} \xi^{2}-\xi^{2}}{|h|} \tau_{h} \nabla u \cdot\left(\int_{0}^{1} \nabla_{x} F(x+(1+s) h, \nabla u(x+h)) \frac{h}{|h|} d s\right) d x .
\end{aligned}
$$

Thanks to (4.4), we get a bound on the absolute value of the second term exactly as we did for the term $I_{3}$. To treat the remaining term, using (4.5) and (4.6), we 
first have

$$
\begin{aligned}
\left|\Phi_{h}\right| & \leq \int_{0}^{1} \frac{\left|\nabla_{x} F(x+s h, \nabla u)-\nabla_{x} F(x+(1+s) h, \nabla u)\right|}{|h|} d s \\
& +\int_{0}^{1} \frac{\left|\nabla_{x} F(x+(1+s) h, \nabla u)-\nabla_{x} F\left(x+(1+s) h, \tau_{h} \nabla u\right)\right|}{|h|} d s \\
& \leq \mu\left[|\mathcal{G}|^{\frac{2 q-2}{q}}+|h|^{-1}\left|\tau_{h} \mathcal{G}-\mathcal{G}\right|\left(|\mathcal{G}|^{\frac{q-2}{q}}+\left|\tau_{h} \mathcal{G}\right|^{\frac{q-2}{q}}\right)\right] .
\end{aligned}
$$

Proceeding as for the term $I_{2}$, we obtain

$$
\left|I_{4}\right| \leq c\left(1+\left\|\xi|h|^{-1}\left(\tau_{h} \mathcal{G}-\mathcal{G}\right)\right\|_{L^{2}}\right) .
$$

Putting all the estimates together, we then get

$$
\left\|\xi \frac{\tau_{h} \mathcal{G}-\mathcal{G}}{h}\right\|_{L^{2}}^{2} \leq c\left(1+\left\|\xi \frac{\tau_{h} \mathcal{G}-\mathcal{G}}{h}\right\|_{L^{2}}\right),
$$

from which we can finally infer

$$
\left\|\frac{\tau_{h} \mathcal{G}-\mathcal{G}}{h}\right\|_{L^{2}(\omega)}^{2} \leq c,
$$

for some constant $c$ depending on $q,\|f\|_{W^{1, p}},\|u\|_{W^{1, q}}$ and the distance between $\omega$ and $\partial \Omega$, but not on $h$. This finally proves the desired result, namely that $\mathcal{G} \in W_{\text {loc }}^{1,2}(\Omega)$.

\subsection{Applications}

We shall now apply Theorem 4.1 to our model equation

$$
-\sum_{i=1}^{d} \partial_{i}\left(\left(\left|\partial_{i} u\right|-\delta_{i}\right)_{+}^{q-1} \frac{\partial_{i} u}{\left|\partial_{i} u\right|}\right)=f,
$$

which corresponds to

$$
F(z)=\left(F_{1}\left(z_{1}\right), \ldots, F_{d}\left(z_{d}\right)\right), \quad \text { with } F_{i}\left(z_{i}\right):=\left(\left|z_{i}\right|-\delta_{i}\right)_{+}^{q-1} \frac{z_{i}}{\left|z_{i}\right|},
$$

in (4.7). We then define

$$
G(z)=\left(G_{1}\left(z_{1}\right), \ldots, G_{d}\left(z_{d}\right)\right), \quad \text { with } G_{i}\left(z_{i}\right):=\left(\left|z_{i}\right|-\delta_{i}\right)_{+}^{\frac{q}{2}} \frac{z_{i}}{\left|z_{i}\right|}
$$

and again we assume that $q \geq 2$. It is immediate to check that condition (4.1) holds and since $F$ does not depend on $x,(4.4),(4.5),(4.6)$ are straightforward. As for (4.2) and (4.3), we have the following. 
Lemma 4.2. Let $F$ and $G$ be defined as above with $q \geq 2$, then for every $(z, w) \in$ $\mathbb{R}^{d} \times \mathbb{R}^{d}$, the following inequalities hold,

$$
|F(z)-F(w)| \leq(q-1)\left(|G(z)|^{\frac{q-2}{q}}+|G(w)|^{\frac{q-2}{q}}\right)|G(z)-G(w)|,
$$

and

$$
(F(z)-F(w)) \cdot(z-w) \geq \frac{4}{q^{2}}|G(z)-G(w)|^{2} .
$$

Proof. First recall (see for instance [16]), that for any $(a, b) \in \mathbb{R}^{2}$, one has

$$
\left.|| a\right|^{q-2} a-|b|^{q-2} b\left|\leq(q-1)\left(|a|^{\frac{q-2}{2}}+|b|^{\frac{q-2}{2}}\right)\right||a|^{\frac{q-2}{2}} a-|b|^{\frac{q-2}{2}} b \mid .
$$

Taking $a=\left(\left|z_{i}\right|-\delta_{i}\right)_{+} z_{i} /\left|z_{i}\right|$ and $b=\left(\left|w_{i}\right|-\delta_{i}\right)_{+} w_{i} /\left|w_{i}\right|$, in the previous inequality, we thus get

$$
\left|F_{i}\left(z_{i}\right)-F_{i}\left(w_{i}\right)\right| \leq(q-1)\left(|G(z)|^{\frac{q-2}{q}}+|G(w)|^{\frac{q-2}{q}}\right)\left|G_{i}\left(z_{i}\right)-G_{i}\left(w_{i}\right)\right|,
$$

and then (4.11) directly follows.

We shall now prove that for every $i$ and $z_{i}, w_{i}$ in $\mathbb{R}$, there holds

$$
\left(F_{i}\left(z_{i}\right)-F_{i}\left(w_{i}\right)\right)\left(z_{i}-w_{i}\right) \geq \frac{4}{q^{2}}\left(G_{i}\left(z_{i}\right)-G_{i}\left(w_{i}\right)\right)^{2},
$$

from which (4.12) will directly follow. First note that (4.14) is straighforward when both $\left|z_{i}\right|$ and $\left|w_{i}\right|$ are less than $\delta_{i}$. If $\left|z_{i}\right|>\delta_{i}$ and $\left|w_{i}\right| \leq \delta_{i}$, we have

$$
\left(F_{i}\left(z_{i}\right)-F_{i}\left(w_{i}\right)\right)\left(z_{i}-w_{i}\right)=\left(\left|z_{i}\right|-\delta_{i}\right)^{q-1}\left(\left|z_{i}\right|-\frac{z_{i}}{\left|z_{i}\right|} w_{i}\right) \geq\left(\left|z_{i}\right|-\delta_{i}\right)^{q}=G_{i}\left(z_{i}\right)^{2} .
$$

As for the case $\left|z_{i}\right|>\delta_{i}$ and $\left|w_{i}\right|>\delta_{i}$, again taking $a=\left(\left|z_{i}\right|-\delta_{i}\right)_{+} z_{i} /\left|z_{i}\right|$ and $b=\left(\left|w_{i}\right|-\delta_{i}\right)_{+} w_{i} /\left|w_{i}\right|$ in the following inequality (for which we again refer to [16])

$$
\left(|a|^{q-2} a-|b|^{q-2} b\right)(a-b) \geq \frac{4}{q^{2}}\left(|a|^{\frac{q-2}{2}} a-|b|^{\frac{q-2}{2}} b\right)^{2},
$$

we get

$$
\frac{4}{q^{2}}\left(G_{i}\left(z_{i}\right)-G_{i}\left(w_{i}\right)\right)^{2} \leq\left(F_{i}\left(z_{i}\right)-F_{i}\left(w_{i}\right)\right)\left(z_{i}-w_{i}\right)-\delta_{i}\left(F_{i}\left(z_{i}\right)-F_{i}\left(w_{i}\right)\right)\left(\frac{z_{i}}{\left|z_{i}\right|}-\frac{w_{i}}{\left|w_{i}\right|}\right) .
$$

We then observe that

$$
\left(F_{i}\left(z_{i}\right)-F_{i}\left(w_{i}\right)\right)\left(\frac{z_{i}}{\left|z_{i}\right|}-\frac{w_{i}}{\left|w_{i}\right|}\right)=\left(\left(\left|z_{i}\right|-\delta_{i}\right)^{q-1}+\left(\left|w_{i}\right|-\delta_{i}\right)^{q-1}\right)\left(1-\frac{z_{i} w_{i}}{\left|z_{i} w_{i}\right|}\right) \geq 0,
$$

which finally proves (4.14). 
If we go back to the variational problem of Beckmann type:

$$
\inf _{\sigma \in L^{p}(\Omega)}\left\{\int_{\Omega} \sum_{i=1}^{d}\left(\frac{1}{p}\left|\sigma_{i}(x)\right|^{p}+\delta_{i}\left|\sigma_{i}(x)\right|\right) d x:-\operatorname{div} \sigma=f\right\}
$$

we then obtain the following Sobolev regularity for the (unique) minimizer.

Corollary 4.3. If $q \geq 2$ and $f \in W_{\mathrm{loc}}^{1, p}(\Omega)$ then the solution $\sigma$ of (4.15) belongs to the Sobolev space $W_{\mathrm{loc}}^{1, r}(\Omega)$, where

$$
r= \begin{cases}2, & \text { if } q=2, \\ \text { any value }<2, & \text { if } q>2 \text { and } d=2, \\ \frac{d q}{d q-(d+q)+2}, & \text { if } q>2 \text { and } d>2 .\end{cases}
$$

Proof. By duality, we know that $\sigma$ is related to any solution of the dual problem $u$ through the componentwise relation

$$
\sigma_{i}=\left(\left|\partial_{i} u\right|-\delta_{i}\right)_{+}^{q-1} \frac{\partial_{i} u}{\left|\partial_{i} u\right|}, \quad i=1, \ldots, d .
$$

Since $u \in W^{1, p}(\Omega)$ is a weak solution of the Euler-Lagrange equation (4.10), it follows from Theorem 4.1 and Lemma 4.2 that the vector field whose components are

$$
\mathcal{G}_{i}:=\left(\left|\partial_{i} u\right|-\delta_{i}\right)_{+}^{\frac{q}{2}} \frac{\partial_{i} u}{\left|\partial_{i} u\right|}, \quad i=1, \ldots, d,
$$

is in $W_{\text {loc }}^{1,2}(\Omega)$ and we obtain the desired result exactly as in [7], by observing that $\sigma_{i}=\left|\mathcal{G}_{i}\right|^{\frac{q-2}{q}} \mathcal{G}_{i}$.

Let us observe that when $d=2$ (the case which is relevant for applications to network congestion), the previous result implies that $\sigma \in L_{\text {loc }}^{s}$ for every $s>1$. As shown recently in [6], this higher integrability result is still valid for $d \geq 2$ and can be proved without appealing to the Sobolev result of Corollary 4.3, by proving directly that any solution of the dual problem has a gradient in $L_{\text {loc }}^{s}$, for every $s>1$. We point out that the proof in [6] requires $f$ to be in $L_{\mathrm{loc}}^{\infty}$, an hypothesis which is not directly comparable with that of Corollary 4.3 .

Remark 4.4. The previous Sobolev result can be easily generalized to equations with weights such as

$$
-\sum_{i=1}^{d} \partial_{i}\left(b_{i}(x)\left(\left|\partial_{i} u\right|-\delta_{i}\right)_{+}^{q-1} \frac{\partial_{i} u}{\left|\partial_{i} u\right|}\right)=f
$$


as well as to non symmetric equations of the form

$$
-\sum_{i=1}^{d} \partial_{i}\left(\left(\partial_{i} u-\delta_{i}\right)_{+}^{q-1}-\left(-\partial_{i} u-\beta_{i}\right)_{+}^{q-1}\right)=f,
$$

and finally to equations of the form (3.4) which both have weights and are nonsymmetric.

Acknowledgements. We thank Filippo Santambrogio for some useful discussions. This work has been supported by the ANR through the projects ANR09-JCJC-0096-01 EVAMEF and ANR-07-BLAN-0235 OTARIE, as well as by the ERC Advanced Grant n. 226234.

\section{References}

[1] L. Ambrosio, Transport equation and Cauchy problem for $B V$ vector fields, Invent. Math. 158 (2004), 227-260.

[2] J.-B. Baillon, G. Carlier, From discrete to continuous Wardrop equilibria, Netw. Heterogenous Media, 7 (2012), 219-241.

[3] M. J. Beckmann, A continuous model of transportation, Econometrica, 20 (1952), 643-660.

[4] M. Beckmann, C. McGuire, C. Winsten, Studies in Economics of Transportation. Yale University Press, New Haven, 1956.

[5] M. Belloni, B. Kawohl, The pseudo $p$-Laplace eigenvalue problem and viscosity solutions as $p \rightarrow \infty$, ESAIM Control Optim. Calc. Var., 10 (2004), $28-52$.

[6] L. Brasco, G. Carlier, On certain anisotropic elliptic equations arising in congested optimal transport: local gradient bounds, preprint (2012) http://cvgmt.sns.it/paper/1890/.

[7] L. Brasco, G. Carlier, F. Santambrogio, Congested traffic dynamics, weak flows and very degenerate elliptic equations, J. Math. Pures Appl., 93 (2010), 652-671.

[8] G. Carlier, C. Jimenez, F. Santambrogio, Optimal transportation with traffic congestion and Wardrop equilibria, SIAM J. Control Optim., 47 (2008), 1330-1350. 
[9] B. Dacorogna, J. Moser, On a partial differential equation involving the Jacobian determinant, Annales del' I.H.P. Anal. non linéaire, 7 (1990), 1-26.

[10] L. De Pascale, L. C. Evans, A. Pratelli, Integral estimates for transport densities, Bull. London Math. Soc., 36 (2004) 36, 383-395.

[11] L. De Pascale, A. Pratelli, Regularity properties for Monge transport density and for solutions of some shape optimization problem, Calc. Var. Partial Differential Equations, 14 (2002), 249-274.

[12] L. De Pascale, A. Pratelli, Sharp summability for Monge Transport density via Interpolation, ESAIM, Control Opt. Calc. Var., 10 (2004), 549-552.

[13] R. J. DiPerna, P.-L. Lions, Ordinary differential equations, transport theory and Sobolev spaces, Invent. Math., 98 (1989), 511-547.

[14] I. Ekeland, R. Temam, Convex analysis and Variational problems, Classics in Applied Mathematics, 28. SIAM, Philadelphia, PA, 1999.

[15] J.-M. Lasry, P.-L. Lions, Mean-Field Games, Japan. J. Math., 2 (2007), 229-260.

[16] P. Lindqvist, Notes on the p-Laplace equation. Report. University of Jyväskylä Department of Mathematics and Statistics, 102. University of Jyväskylä, Jyväskylä, 2006, available at http://www. math.ntnu.no/ lqvist/

[17] J. Moser, On the volume elements on a manifold, Trans. Am. Math. Soc., 120 (1965), 286-294.

[18] F. Santambrogio, Absolute continuity and summability of transport densities: simpler proofs and new estimates, Calc. Var. Partial Differential Equations, 36 (2009), 343-354.

[19] J. G. Wardrop, Some theoretical aspects of road traffic research, Proc. Inst. Civ. Eng. 2 (1952), 325-378. 